\title{
Social system as complex networks
}

\author{
Gourab Ghoshal · Giuseppe Mangioni · \\ Ronaldo Menezes $\cdot$ Julia Poncela-Casanovas
}

Published online: 21 November 2014

(C) Springer-Verlag Wien 2014

In the last decade we have seen the emergence of a new inter-disciplinary field of research focusing on the study of networks which are large, dynamic, multi-layered, and structured. A network is a mathematical object consisting of a set of vertices (or nodes) that are connected to each other by edges. This simple description corresponds to a wide array of systems in the real world, ranging from technological ones such as the Internet and World Wide Web, biological networks such as that of connections of the nervous systems, food webs, or protein interactions, infrastructural systems such as networks of roads, airports or the power-grid, to patterns of social and professional relationships such as friendship, sex partners, network of Hollywood actors, co-authorship networks and many more. The field of Complex Networks is helping us to uncover the features of numerous complex phenomena such as the spread of diseases, protein interactions, social relationships, just to name a few.

\footnotetext{
G. Ghoshal

Department of Physics, Biology and Computer Science, Center for Complex Network Research, Northeastern University, Boston, MA, USA

G. Mangioni $(\square)$

Department of Electrical Electronics and Computer Engineering, University of Catania, Catania, Italy

e-mail: giuseppe.mangioni@dieei.unict.it

R. Menezes

BioComplex Laboratory, Department of Computer Sciences, Florida Institute Technology, Melbourne, FL, USA

e-mail: rmenezes@cs.fit.edu

\section{J. Poncela-Casanovas}

Amaral Laboratory, Department of Chemical and Biological Engineering, Northwestern University, Evanston, IL, USA e-mail: poncela@northwestern.edu
}

This special issue brings together a collection of cuttingedge research in the field highlighting the strong interconnections between complex networks and social systems.

The article "Social capitalists on Twitter: detection, evolution and behavioral analysis" by Nicolas Dugue' and Anthony Perez proposes an algorithm to detect social capitalists, a special class of users who post on the Twitter network. They also study the evolution of such users between 2009 and 2013 examining behavorial trends based on the semantic content of the tweets.

Many real-world networks are very large and for this reason there is considerable interest in methods that can analyze them in a efficient way. The article "Scalable Graph Clustering with Parallel Approximate PageRank" by Bryan Perozzi, Christopher McCubbin and J.T. Halbert proposes a parallel technique to create Approximate PageRank vectors and local clusters by using parallel processing techniques based on Pregel and MapReduce.

The article entitled "On the Computation of Entropy Production in Stationary Social Networks" by Tobias Hofeld, Valentin Burger, Haye Hinrichsen, Matthias Hirth and Phuoc Tran-Gia, introduces a quantity called entropy production as a measure to study stationarity in social networks. They uncover a relation between entropy production and the traditional structural metrics used to study social networks. Moreover, the authors extend their analysis to the realm of real networks by employing techniques based on Bayesian inference.

Several networks are characterized by the presence of a community structure that can be used as a key element to explain many behavioral patterns. In the article "Multiego-centered communities in practice" by Maximilien Danisch, Jean-Loup Guillaume and Benedicte Le Grand a framework is provided to uncover the ego-centered 
community structure of an extant node in a network. It is based on the search of irregularities in the decrease on the values of a proximity measure. They show an application of their approach to a set of data extracted from Wikipedia.

The article "Using Metrics from Network Science to Assess Particle Swarm Optimizers" by Marcos OliveiraJunior, Carmelo Bastos-Filho and Ronaldo Menezes presents an application of a metric used in complex networks field to improve the performance of a Particle Swarm Optmizer. Their proposal is based on the definition of an influence graph used to assess the dynamics of the swarm. The efficacy of the proposed approach is tested on a set of well-known benchmark function.

The advent of interactive online social networks poses new interesting challenges. The article "The Social Distributional Hypothesis-A pragmatic proxy for homophily in online social networks" by Folke Mitzlaff, Martin Atzmueller, Andreas Hotho and Gerd Stumme presents the study of the social distribution hypothesis-users with similar interaction characteristics tend to be semantically related. This hypothesis has been investigated by the authors by considering a range of user interactions in Twitter, Flicker and BibSonomy.

Acknowledgments Great thanks are first given to SNAM and its Editor-in-Chief, Reda Alhajj who kindly offered the publication of this special issue. We would also like to thank Robert Tolksdorf from Freie Universitaet Berlin, Germany and his group who greatly helped us with hosting and organizing CompleNet2013. In addition, we would like to thank all the reviewers for their tireless reviewing efforts and valuable feedback. Finally we thank the authors for their interest in and submissions to this special issue. 